3 Bruyn GW. Huntington's Chorea: differential diagnosis. In: Vinken PJ, Bruyn GW, eds Handbook of Clinical Neurology. Vol 6. Amsterdam: North-Holland, 1968:354.

4 Trelles JO, Trelles L. Cysticercosis of the Nervous System. In: Vinken PJ, Bruyn GW, eds Handbook of Clinical Neurology. Vol 35 Amsterdam: North-Holland, 1978:291-320.

\section{Glycolytic enzymes in the CSF as tumour markers}

Sir: In agreement with Twijnstra and colleagues $^{1}$ I find measurements of glycolytic enzymes in cerebrospinal fluid (CSF) such as lactate dehydrogenase (LDH) or phosphoglucoseisomerase (PHI) easy to perform, readily available and economical. A drawback in the detection of meningeal metastases is their limited specificity. The following suggestions may help to reach a greater specificity.

Although the authors have considered the possible influence of age and sex on enzyme activity in the CSF, they have apparently not taken into account the state of the blood brain barrier (BBB) and the enzyme levels in the blood. In evaluating plasma protein concentrations in CSF such as IgG, it is common practice nowadays to correct the CSF value for the serum derived fraction. A similar approach was recently undertaken for the estimation of another tumour marker, carcinoembryonic antigen in CSF. ${ }^{2}$

$\mathrm{CSF} /$ serum ratios range from $1 / 230$ for albumin to $1 / 500$ for IgG. The permeability of the BBB for these plasma proteins is governed by their molecular radii. ${ }^{2}$ By analogy, for LDH, an enzyme with $140000 \mathrm{D}$ molecular weight, one would expect a quotient of about $1 / 360$. With a serum value of $240 \mathrm{U} / 1$ (the upper limit of normal with standardised methods) one then arrives at $240 / 360=0.7$ $\mathrm{U} / \mathrm{l}$ for the LDH activity in the CSF. However, the normal value given by Twijnstra $e t$ $a l^{1}$ lies at $10 \mathrm{U} / 1$. Therefore, in great contrast to plasma proteins, only approximately $7 \%$ of the LDH activity in CSF originates from the blood under normal conditions. Accordingly corrections for serum LDH may appear unnecessary. While this is probably true for controls with normal $\mathrm{LDH}$ and intact BBB, it is different for cancer patients. Meningeal affection often causes considerable impairment of the BBB and in the presence of systemic metastases LDH serum activity may be elevated several fold Erroneously high CSF values may result especially when both conditions coincide. For PHI a correction formula analogous to Tourtellotte's calculation of the IgG synthesis rate has been proposed. ${ }^{3}$

It would be interesting to know if the three control persons with pathological CSF values above $26 \mathrm{U} / 1$ in Twijnstra's patients ${ }^{1}$ displayed elevated serum LDH and/or BBB disturbances. Likewise the observed aug mentation of CSF LDH in older subjects may be connected to the greater permeability of the BBB for people aged over 60 years. In addition, simultaneous measurement of CSF and serum enzyme activities often reveal a beneficial effect of the CNS-directed therapy, when at the same time the systemic cancer cannot be controlled. ${ }^{3}$

U WURSTER CSF Laboratory, Department of Neurology and Neurophysiology. Medical School, Hannover, PO Box 610180 D-3000 Hannover 61 ,

\section{References}

1 Twijnstra A, van Zanten AP, Hart AAM Ongerboer de Visser BW. Serial lumbar and ventricle cerebrospinal fluid lactate dehydrogenase activities in patients with leptomeningeal metastases from solid and haematological tumours. J Neurol Neurosurg Psychiatry 1987;50:313-20.

2 Reiber HO, Jacobi C, Felgenhauer K. Sensitive quantitation of carcinoembryonic antigen in cerebrospinal fluid and its barrier-dependent differentiation. Clin Chim Acta 1986;156: 259-70.

3 Wurster U, Engelhardt P. Phosphohexoseisomerase und B-Glukuronidase im Liquor cerebrospinalis bei Hirnmetastasen. Aktuelle Onkologie 1984;13:210-7.

\section{Shoulder abduction fatiguability}

Sir: I read with considerable interest the recent article by Nicklin et al entitled Shoulder abduction fatiguability. ${ }^{1}$ Having previously read and admired the work of two of the authors, I was encouraged to observe that their work continues in the application of hand-held dynamometry to assess the neurological patient. I have, nevertheless, several concerns with what the authors have recently presented in this journal.

My chief concern is their apparent failure to take into account the influence of gravity during testing. By either testing shouldero abduction against gravity and not correcting for its influence or mixing the results of testsen performed against gravity (in sitting) with $\frac{\bar{\sigma}}{6}$ those of tests performed with gravity eliminated (when supine) a potential source ofs error was introduced. Winter et al calculatedo that a failure to correct for gravity effects resulted in an absolute percentage difference of 2.4 (range -6.5 to 26.0 ) for knee exten :sion. $^{2}$ The potential error associated with a failure to take gravity into account can be illustrated as follows: Suppose a subject'sc arm places 15 Newtons of force on a dynamometer, at its point of application. That is, with the arm abducted to $90^{\circ}$ and the elbow气 flexed to $90^{\circ}$ but resting on the dyna-n mometer, a force of 15 Newtons is registered"s because of gravity. Next, suppose that the seated subject generates 140 Newtons of $\rightarrow$ abduction force when tested as suggested by $\vec{\omega}$ the authors. A $6.0 \%$ decline in this force? over a series of 10 contractions would be 8.47 Newtons. This value, 8.4 Newtons, is only? $5.4 \%$ of 155 Newtons, the actual force pro-duced (140 Newtons +15 Newtons to holdthe arm against gravity). Thus, the fatigue index is decreased by $(6 \cdot 0-5 \cdot 4) / 6 \cdot 0=10 \% \mathrm{G}$ by including the weight of the arm. Now us assume that the same subject is affected을 with a disorder that renders her weak. Gray-ity still results in 15 Newtons of force frबm $Z$ the arm. The subject now generates $30 \mathrm{New}-\mathrm{O}$ tons of force while seated. A $6.0 \%$ decline्gn this force equals 1.8 Newtons, which is $4.0 \%$ of 45 Newtons (30 Newtons + 15 Newt $\overline{\text { pis }}$ s to hold the arm against gravity). Thus, the fatigue index is decreased by $(6.0-4 \cdot 0) / 6.000$ $=33.3 \%$ by including the weight of the. arm. Granting that this is a highly hypothetical situation; the error resulting from a failure to correct for gravity, particularly in a weak arm, could be quite serious.

My second concern is with the muscle $\frac{0}{\Phi}$ group selected by the authors for theire study. Although good reasons probably $\overrightarrow{\vec{\sigma}}$ exist for the authors' choice of the shoulder 3 abductors, these muscles are probably more? difficult than some others to test accurately. In a study in which I tested supine subjects I found that forces obtained during repeated tests of shoulder abduction, unlike forces? obtained during most other actions, differed $\frac{0}{3}$ significantly from one another. ${ }^{3}$ I have observed that subjects tend to flex the trunko toward the contralateral side during shoul- $₹$ der abduction testing. This tendency, whicho is particularly apparent when subjects are tested in sitting, magnifies actual force prod $\frac{D}{2}$ uction. Even the subject in the authors' fig 1 . seems to be flexed to the right during testing $N$ 